case study presentation seeks to propose alternative methods for public health emergency response in emergency management, through lessons learned and the development of the Emergency Operation Center (EOC) planning tools.

Background: Public health incidents pose a challenge for emergency management agencies because they do not follow the same "stand up" and "stand down" style of natural disasters or catastrophic incidents. Typically, public health incidents begin with more ambiguity than emergency managers usually encounter in other types of naturally occurring or manmade incidents. These incidents require technical, regulatory and scientific expertise that involves various non-health stakeholders for general consequence management.

Methods: NYC is currently developing a draft of Public Health Response protocols and tools that integrate SMEs from nonhealth, Medical and/or Human Services agencies based on preparedness and response activities, hot washes, and After-Action Reports. With the goal of connecting expertise of particular ESFs and task forces, such as waste management in non-clinical settings, public information, community engagement, and consequence management, this presentation will allow focus group members/conference attendees to think through select public health incidents (based on NYC's case studies), that require significant non-public health and medical stakeholders.

Results: Research/field testing is still in progress but preliminary information may be available by Spring 2017.

Conclusion: More research/field testing is needed to formally integrate public health emergency management into the current ESF mechanisms used in local emergency management agency EOCs.

Prehosp Disaster Med 2017;32(Suppl. 1):s115-s116

doi:10.1017/S1049023X17003302

\section{Maternity Care Model during a Natural Disaster or Humanitarian Emergency Setting in Rural Pakistan Humaira Maheen}

School of Health and Social Development, Deakin University, Melbourne/Australia

Study/Objective: To propose a maternity care model for natural disaster or humanitarian setting in rural Pakistan, by using its existing Health Human Resource (HRH).

Background: Pakistan has been severely affected by a number of natural disasters, as well as humanitarian emergencies in the last decade. There are more than 100,000 health workers (including community and facility based midwives) in rural Pakistan which are local, trained, and most of them possess a good relationship with their community. There is a need to establish effective strategies, to utilize their services as frontline maternity care workers during emergency and conflict situations.

Methods: A mixed methods study was conducted in floodaffected villages of Sindh, which included 60 interviews (15 from women, 25 from Community Health workers, and 20 from key informants) and a survey with 669 women about their preferences of maternity care providers.

Results: In the absence of obstetric facilities in relief camps and a functional referral system, $91.2 \%$ women gave birth in temporary shelters with the help of a traditional birth attendant (Dai) with no clean physical space available to birth. Community health workers were not involved in disaster related activities. A clean delivery kit, dignity kit, and contraception were not available at relief camps.

Conclusion: The existing health workers are recommended to be engaged at different stages of a natural disaster (preparedness, early warning, and response). The capacity building of health workers and district health officials on emergency management is highly recommended. District health authorities should collaborate with Humanitarian Health Cluster at predisaster time to optimal utilization of logistic, financial and human resources. A well equipped "birthing station" and "women friendly spaces" are recommended in each camp. Referral systems should be strengthened whereby all laboring women with complications, can be timely transferred (transportation provided) to the nearest rural health facility where they will be assisted by the Emergency Obstetric Care (EMoC) trained staff.

Prehosp Disaster Med 2017;32(Suppl. 1):s116

doi:10.1017/S1049023X17003314

The Social Impact of Terrorism on Civilian Populations Lessons Learned from Decades of Terrorism in Israel and Abroad

Maya Siman-Tov ${ }^{1}$, Moran Bodas ${ }^{2}$, Kobi Peleg ${ }^{3}$

1. Trauma And Emergency Medicine, Gertner, Ramt-Gan/Israel

2. Tel-Aviv University, Tel Aviv/Israel

3. Disaster Management \& Injury Prevention, Tel-Aviv University, Tel Aviv/Israel

Study/Objective: This study considers the socio-psychological implications of terrorism, which are sometimes neglected in preparedness plans.

Background: One of the main concerns for public safety, shared by many countries, is the fear of terrorism; yet, far fewer lives are lost yearly due to terrorism as opposed to other forms of trauma, such as traffic accidents. Why does terrorism receive so much attention and incite such intense apprehension? Perhaps, terrorism "packs a different punch," one that goes far beyond the number of injuries and fatalities. Terrorism's main goal is to disrupt ordinary life, fostering fear and helplessness in the population.

Methods: Using Israeli experiences as a case study, this study briefly reviews four points of connection between terrorism and its psychological and social legacies: the sociopolitical aspects of terrorism, the unexpected nature of terrorism, normalization of terrorism and public resilience, and social aspects of medical care for terror-related injuries.

Results: The Israeli experience suggests preparedness plans should include planning for the socio-psychological effects of terrorism, on targeted populations, and may, in certain contexts, use Israeli approaches as a model.

Conclusion: Experience gained in Israel and elsewhere can set the stage for an appropriate response plan, striving not only for preparedness but also resilience. Efforts should be made to advance local capabilities, response plans, and resilience by 